Abstract OC-056 Table 1 Trends in the prevalence of taking potentially damaging and protective drugs

\begin{tabular}{lll}
\hline DRUG & OR (95\%Cl) & P-Value \\
\hline Aspirin & $1.025(1.002-1.048)$ & 0.030 \\
Other antithrombotic drugs & $1.089(1.057-1.122)$ & $<0.001$ \\
NSAIDs & $1.014(0.985-1.043)$ & 0.35 \\
Beta blockers & $1.086(1.054-1.118)$ & $<0.001$ \\
ACE inhibitors & $1.136(1.100-1.173)$ & $<0.001$ \\
SSRI & $1.048(1.009-1.088)$ & 0.015 \\
Statins & $1.168(1.131-1.207)$ & $<0.001$ \\
Proton pump inhibitors & $1.126(1.102-1.152)$ & $<0.001$ \\
\hline
\end{tabular}

Conclusion Patients with UGIB presented with progressively increasing comorbidity over the 14-year period 1996-2010. The use of both potentially damaging and protective drugs also increased. Raw 30-day mortality was unchanged over the same period, but decreased significantly with time when adjusted for comorbidity. These observations might have clinical implications.

Disclosure of Interest A. Taha Consultant for: Horizon Pharma USA; Vifor Pharma UK, E. Saffouri: None Declared, C. McCloskey: None Declared, T. Craigen: None Declared, W. Angerson: None Declared

\section{OC-057 THE "SPEEDBOAT": A NEW MULTI-MODALITY INSTRUMENT FOR ENDOSCOPIC RESECTION IN THE GASTROINTESTINAL TRACT}

doi:10.1136/gutjnl-2013-304907.056

1,"Z P Tsiamoulos, ${ }^{2} \mathrm{C}$ Hancock, ${ }^{3} \mathrm{~L}$ A Bourikas, ${ }^{4} \mathrm{P}$ D Sibbons, ${ }^{5} \mathrm{~B}$ P Saunders. 'Wolfson Unit for Endoscopy, St Mark's Hospital and Academic Institute, Harrow, Middlesex; ${ }^{2}$ Creo Medical Ltd, Bangor University, Bangor, UK; ${ }^{3}$ Department of Gastroenterology, Univesity Hospital of Heraklion, Crete, Greece; ${ }^{4}$ Division of Surgical Sciences, Northwick Park Institute of Medical Research; '5olfson Unifor Endoscopy, St Mark's Hospital and Academic Institute, Harrow, Middlesex, UK

Introduction Large sessile or flat intestinal lesions $>2 \mathrm{~cm}$ are optimally removed en-bloc for more accurate histology and for completeness of resection. Current submucosal dissection devices are technically challenging to use resulting in long and sometimes incomplete procedures with a high risk of major complication. We describe, for the first time, a simple to use, multimodality endoscopic device ("Speedboat") for wide-field, en-bloc mucosal resection.

Methods The 'Speedboat' cuts in forward, lateral and oblique planes using bipolar radio frequency (RF) cutting, provides haemostasis with microwave coagulation and incorporates a retractable needle for submucosal injection and tissue irrigation. The instrument blade has an insulated 'hull' to prevent thermal injury and the device catheter is partially torque stable allowing rotation and orientation of the hull to protect the underlying muscularis propria. The electrosurgical generator is comprised of 2 power sources, one operating at $400 \mathrm{KHz}(\mathrm{RF})$ and the other at $5.8 \mathrm{GHz}$ (microwave).

Speedboat submucosal dissection technique (SSD) was performed and video recorded on 3 consecutive $60 \mathrm{~kg}$ pigs. Mucosal areas to be resected were marked prior to submucosal injection. The mucosa was then circumferentially incised and resected by SSD. The time taken to complete resection, complications encountered and power settings used were recorded. Immediately after the procedure, the animals were euthanized, and the resection defects measured and assessed histologically.

Results Eight consecutive resections were performed (2 per ani$\mathrm{mal}), 7$ in the colorectum and 1 in the antrum of the stomach. The median time to complete a resection was 37 minutes range (30-60 minutes) using RF cutting $24 \mathrm{~W}$, voltage circa $300 \mathrm{Vrms}$. Median defect size (longest diameter) was $53.5 \mathrm{~mm}$, range $40-80 \mathrm{~mm}$. Microwave coagulation was applied for either minor bleeding or visible vessels on 32 occasions (mean energy 7.5 W). An endoclip was used once to control arteriolar bleeding but no other haemostatic device was required. There were no perforations and histology showed an intact and viable muscle layer with some remaining submucosa in all cases.

Conclusion This initial evaluation of the "Speedboat" suggests that it facilitates rapid and safe en-bloc mucosal resection in the colorectum and gastric antrum.

Disclosure of Interest Z. Tsiamoulos Grant/Research Support from: Creo Medical Ltd, Consultancy agreement, C. Hancock Shareholder of: Creo Medical Ltd, L. Bourikas: None Declared, P. Sibbons: None Declared, B. Saunders Consultant for: Creo Medical Ltd, Consultancy and Intellectual Property agreement

\section{Small bowel and nutrition free papers}

\section{OC-058 BILE ACID MALABSORPTION: "THE UNUSUAL SUSPECT". A VERY FREOUENT CAUSE FOR DIARRHOEA AFTER TREATMENT FOR MANY DIFFERENT CANCER TYPES}

doi:10.1136/gutjnl-2013-304907.057

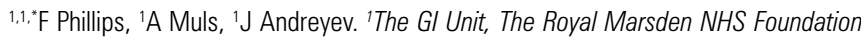
Trust, London \& Surrey, UK

Introduction Approximately 16,000 patients per year are left with a chronic change in bowel function and gastrointestinal symptoms following treatment for cancer. This can have a significant impact upon quality of life and even compromise ongoing cancer therapy. Previous small studies focusing mainly on patients with gynaecological cancers suggest that bile acid malabsorption (BAM) may be an important cause of radiotherapyinduced diarrhoea. This retrospective study aims to elucidate the prevalence of BAM in patients referred to a specialist gastroenterology clinic which treats patients with consequences of cancer treatments.

Methods Patients referred to our clinic are managed using a peer reviewed algorithm. If they report diarrhoea, defined as Bristol Stool Chart type 6-7, a routine referral for a SeHCAT (75-selenium homocholic acid taurine) scan is made to exclude BAM. BAM is diagnosed when the SeHCAT 7 day retention is $<15 \%$ and is graded mild (10-15\%), moderate (5-10\%) and severe $(<5 \%)$.

Results Between 2009-2012, 262 patients were referred for a SeHCAT scan. 139 (53.1\%) were male (median age 66, range 32-89) and 123 (46.9\%) female (median age 61, range 20-86). $79(37 \%)$ patients had been treated for gynaecological, 94 (44\%) for urological, $41(19 \%)$ for anorectal and $30(11 \%)$ for upper gastrointestinal tract cancers. A further $18(7 \%)$ were treated for other cancer types (breast, lung and haematological). BAM was diagnosed in 24 patients $(25 \%)$ treated for urological cancers, with $17 \%(n=4)$ having severe BAM. 46 patients $(58 \%)$ treated for gynaecological cancers had BAM, severe in $57 \%(\mathrm{n}=26)$. BAM was diagnosed in 22 patients (54\%) treated for anorectal cancers, with $55 \%$ having severe BAM ( $\mathrm{n}=12)$. In 15 patients $(50 \%)$ treated for upper GI cancers, the majority $(73 \%)$ had severe BAM $(n=11)$. For patients with other cancer types, BAM was diagnosed in 8 patients (44\%) with severe BAM in 2 of these (25\%).

Conclusion The frequency of severe bile acid malabsorption and its impact on quality of life is poorly appreciated amongst those who treat cancer. BAM is a common problem in patients treated for a wide variety of cancers with a number of different treatment modalities. Given the effectiveness of dietary fat manipulation combined with a new generation of bile acid sequestrants, SeHCAT scanning should be a first line in investigating patients with diarrhoea after cancer therapies.

Disclosure of Interest None Declared 